\title{
Highlighting recognition, management, and treatment of neural tube defects at various stages of development
}

\author{
Darcie Kiddoo, MD \\ University of Alberta, Edmonton, AB, Canada
}

Cite as: Can Urol Assoc J 2017;11 (1-2Suppl1):S87. http://dx.doi.org/10.5489/cuaj.4382

See related article on page $\mathrm{S} 81$.

$\mathrm{N}$ eural tube defects in children result in a significant burden from the medical sequelae to the impact in quality of life. The focus years ago was on protecting kidney function and improving lifespan. As care has improved, this has shifted to quality of life for patients. We now face the challenge of transitioning children to the adult world. It is, therefore, increasingly important for all urologists to understand the management strategies used in the current care of children with this condition.

Dr. Metcalfe's review highlights the important steps in recognition, management, and treatment at stages of development. The initial care for infants to preschoolers is on health and prevention of complications. He describes expectant vs. proactive care, highlighting the success of proactive care at his centre. This includes early adoption of clean intermittent catheterization $(\mathrm{CIC})$ and monitoring with urodynamics. This prevents urinary tract infections (UTIs) and identifies bladder changes early. Recognition of bladder changes allows early introduction and maximization of anticholinergic medication.

In elementary school and adolescence, most families focus on their desire for continence. Interventions include optimizing medication and catheterization, as well as surgical options. Most surgical interventions, including onabotulinumtoxinA injections, bladder augmentation, and catheterizable channels require motivated patients for success. Dr. Metcalfe also highlights the need to work with the families to ensure the best treatment option for their personal situation. If reliability is a concern, opting for safe alternatives with incontinent diversions are preferred.

As children enter adulthood, new issues arise. At this time, health can deteriorate and the lack of support can prove to be a problem. The importance of adult urologists who can provide care becomes critical. Through this review, Dr. Metcalfe summarizes optimum care for patients as they travel through the system in time. With increased knowledge, we can continue to improve on health and quality of life even outside of multidisciplinary clinics.

Competing interests: The author reports no competing personal or financial interests.

This paper has been peer-reviewed.

Correspondence: Dr. Darcie Kiddoo, University of Alberta, Edmonton, AB, Canada; dkiddoo@ualberta.ca 